\title{
Giant Fold Gastritis Induced by Epoprostenol Infusion in Patients With Pulmonary Arterial Hypertension
}

\author{
Yohei Miura, MD; Masaharu Kataoka, MD; Tomohiro Chiba, MD; \\ Takumi Inami, MD; Hideaki Yoshino, MD; Toru Satoh, MD
}

\begin{abstract}
Background: Epoprostenol infusion is the strongest and most convincing therapeutic strategy for severe pulmonary arterial hypertension (PAH). This study investigated the gastrointestinal side effects of epoprostenol.
\end{abstract}

\begin{abstract}
Methods and Results: The study group of 12 patients treated with epoprostenol (epoprostenol group) and 4 patients without epoprostenol (control group) underwent stomach barium examination, which revealed that the prevalence of giant fold gastritis was significantly higher in the patients treated with epoprostenol ( $75 \%$ in epoprostenol group vs. $0 \%$ in control group; $P=0.019)$.
\end{abstract}

Conclusions: Giant fold gastritis may be an important side effect of epoprostenol infusion.

Key Words: Epoprostenol; Giant fold gastritis; Pulmonary arterial hypertension; Stomach barium examination

$\mathbf{E}$ poprostenol infusion is the strongest and most convincing therapeutic strategy for severe pulmonary arterial hypertension (PAH), ${ }^{1}$ but has some potential side effects such as thrombocytopenia and disturbed thyroid function. ${ }^{2}$ We found giant fold gastritis as a pathological finding in some deceased patients with idiopathic or heritable PAH who had been treated with epoprostenol. Therefore, we conducted a clinical study to investigate the prevalence of giant fold gastritis in idiopathic or heritable PAH patients treated with epoprostenol.

We enrolled 12 PAH patients treated with epoprostenol (epoprostenol group) and 4 untreated PAH patients (control group). Inclusion criteria were female patients aged 20-60 years with idiopathic or heritable PAH who agreed to participate in this study, and exclusion criteria were those who disagreed. All enrolled patients gave informed consent, and the hospitals' institutional review board approved the study protocol and the analysis of data. All patients underwent stomach barium examination. At the timing of this examination, although the median [interquartile range (IQR)] albumin level was significantly lower in the epoprostenol group (4.0 (IQR 3.7-4.4) $\mathrm{g} / \mathrm{dL}$ in

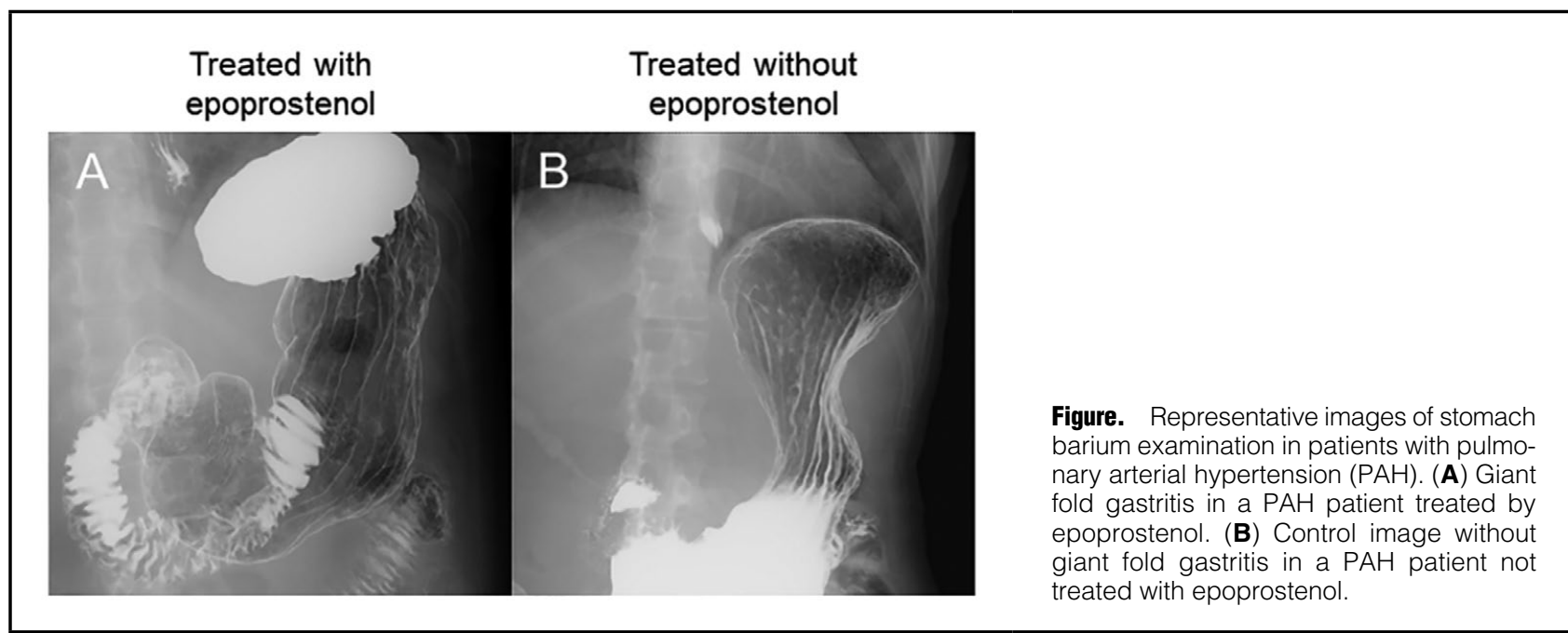

Received April 2, 2018; revised manuscript received July 12, 2018; accepted August 7, 2018; released online August 22,2018 Time for primary review: 84 days

Division of Cardiology, Second Department of Internal Medicine (Y.M., T.I., H.Y., T.S.), Department of Pathology (T.C.), Kyorin University School of Medicine, Tokyo; Department of Cardiology, Keio University School of Medicine, Tokyo (M.K.), Japan

Mailing address: Masaharu Kataoka, MD, Department of Cardiology, Keio University School of Medicine, 35 Shinanomachi, Shinjuku-ku, Tokyo 160-8582, Japan. E-mail: m.kataoka09@keio.jp

ISSN-1346-9843 All rights are reserved to the Japanese Circulation Society. For permissions, please e-mail: cj@j-circ.or.jp 


\begin{tabular}{|c|c|c|c|c|c|c|c|c|}
\hline $\begin{array}{c}\text { Group / } \\
\text { Age } \\
\text { (years) }\end{array}$ & Sex & $\begin{array}{c}\text { BMI } \\
\left(\mathrm{kg} / \mathrm{m}^{2}\right)\end{array}$ & $\begin{array}{l}\text { Duration of } \\
\text { epoprostenol } \\
\text { use (years) }\end{array}$ & $\begin{array}{l}\text { Dose of } \\
\text { epoprostenol } \\
\text { (ng/kg/min) }\end{array}$ & $\begin{array}{l}\text { Giant fold } \\
\text { gastritis }\end{array}$ & $\begin{array}{l}\text { Digestive } \\
\text { symptoms }\end{array}$ & $\begin{array}{l}\text { PAH-specific drugs } \\
\text { (dose/day) }\end{array}$ & $\begin{array}{l}\text { Stomach } \\
\text { medicines } \\
\text { (dose/day) }\end{array}$ \\
\hline \multicolumn{9}{|c|}{ Epoprostenol group } \\
\hline 33 & $\mathrm{~F}$ & 19 & 3 & 38 & + & - & $\begin{array}{l}\text { Macitentan } 10 \mathrm{mg}+ \\
\text { Tadalafil } 40 \mathrm{mg}\end{array}$ & - \\
\hline 35 & $\mathrm{~F}$ & 24 & 5 & 34 & + & - & Macitentan $10 \mathrm{mg}$ & - \\
\hline 38 & $\mathrm{~F}$ & 26 & 9 & 52 & + & Heart burn & $\begin{array}{l}\text { Bosentan } 250 \mathrm{mg}+ \\
\text { Tadalafil } 40 \mathrm{mg}\end{array}$ & - \\
\hline 47 & $\mathrm{~F}$ & 16 & 11 & 55 & + & Diarrhea & $\begin{array}{l}\text { Bosentan } 250 \mathrm{mg}+ \\
\text { Sildenafil } 60 \mathrm{mg}\end{array}$ & - \\
\hline 47 & $\mathrm{~F}$ & 18 & 9 & 26 & + & - & $\begin{array}{l}\text { Bosentan } 250 \mathrm{mg}+ \\
\text { Sildenafil } 60 \mathrm{mg}\end{array}$ & - \\
\hline 48 & $\mathrm{~F}$ & 22 & 5 & 113 & + & Diarrhea & $\begin{array}{c}\text { Macitentan } 10 \mathrm{mg}+ \\
\text { Riociguat } 7.5 \mathrm{mg}\end{array}$ & $\begin{array}{l}\text { Famotidine } \\
20 \mathrm{mg}\end{array}$ \\
\hline 51 & $\mathrm{~F}$ & 21 & 7 & 31 & + & - & $\begin{array}{l}\text { Bosentan } 250 \mathrm{mg}+ \\
\text { Tadalafil } 40 \mathrm{mg}\end{array}$ & - \\
\hline 51 & $\mathrm{~F}$ & 21 & 10 & 46 & + & Diarrhea & $\begin{array}{l}\text { Ambrisentan } 5 \mathrm{mg}+ \\
\text { Sildenafil } 60 \mathrm{mg}\end{array}$ & - \\
\hline 52 & $\mathrm{~F}$ & 19 & 2 & 32 & + & Diarrhea & $\begin{array}{l}\text { Ambrisentan } 5 \mathrm{mg}+ \\
\text { Tadalafil } 40 \mathrm{mg}\end{array}$ & $\begin{array}{c}\text { Esomeprazole } \\
20 \mathrm{mg}\end{array}$ \\
\hline 37 & $\mathrm{~F}$ & 18 & 2 & 110 & - & Diarrhea & $\begin{array}{l}\text { Macitentan } 10 \mathrm{mg}+ \\
\text { Tadalafil } 40 \mathrm{mg}\end{array}$ & - \\
\hline 43 & $\mathrm{~F}$ & 18 & 12 & 28 & - & - & $\begin{array}{c}\text { Ambrisentan } 10 \mathrm{mg}+ \\
\text { Riociguat } 7.5 \mathrm{mg}\end{array}$ & - \\
\hline 49 & $\mathrm{~F}$ & 16 & 15 & 55 & - & Diarrhea & $\begin{array}{l}\text { Macitentan } 10 \mathrm{mg}+ \\
\text { Sildenafil } 60 \mathrm{mg}\end{array}$ & - \\
\hline \multicolumn{9}{|c|}{ Control group } \\
\hline 29 & $\mathrm{~F}$ & 33 & \multicolumn{2}{|c|}{ No epoprostenol use } & - & Melena & $\begin{array}{l}\text { Macitentan } 10 \mathrm{mg}+ \\
\text { Riociguat } 7.5 \mathrm{mg}\end{array}$ & - \\
\hline 31 & $\mathrm{~F}$ & 19 & & & - & - & $\begin{array}{l}\text { Macitentan } 10 \mathrm{mg}+ \\
\text { Riociguat } 7.5 \mathrm{mg}\end{array}$ & - \\
\hline 37 & $\mathrm{~F}$ & 22 & & & - & - & $\begin{array}{c}\text { Macitentan } 10 \mathrm{mg}+ \\
\text { Sildenafil } 60 \mathrm{mg}+ \\
\text { Beraprost } 360 \mu \mathrm{g}\end{array}$ & - \\
\hline 62 & $\mathrm{~F}$ & 19 & & & - & - & $\begin{array}{c}\text { Macitentan } 10 \mathrm{mg}+ \\
\text { Riociguat } 7.5 \mathrm{mg}\end{array}$ & - \\
\hline
\end{tabular}

Giant fold gastritis was diagnosed by radiology specialists when the wall thickening was $\geq 4 \mathrm{~mm}$ with dilatation of the stomach after use of a foaming agent. BMI, body mass index; $\mathrm{PAH}$, pulmonary arterial hypertension.

epoprostenol group vs. 4.5 (IQR $4.4-4.6$ ) g/dL in control group, $\mathrm{P}=0.045)$, hemodynamic and other biomarkers were not significantly different between the 2 groups [mean pulmonary arterial pressure, 27 (IQR 21-32) $\mathrm{mmHg}$ vs. 35 (IQR 28-40) $\mathrm{mmHg}, \mathrm{P}=0.078$; pulmonary vascular resistance, 4.2 (IQR 2.8-5.8) Wood units vs. 6.2 (IQR 4.9-7.0) Wood units, $\mathrm{P}=0.078$; cardiac output, 4.7 (IQR 4.0-5.4) $\mathrm{L} / \mathrm{min}$ vs. 4.5 (IQR 3.5-5.4) L/min, $\mathrm{P}=0.314$; B-type natriuretic peptide, 20.8 (IQR 7.7-42.8) pg/mL vs. 27.8 (IQR 6.6-60.4) $\mathrm{pg} / \mathrm{mL}, \mathrm{P}=0.973$; in epoprostenol vs. control group, respectively]. Medication with regard to other PAH-specific drugs and gastric medicines were not significantly different between the 2 groups by Fisher's exact test.

Stomach barium examination revealed that the prevalence of giant fold gastritis was significantly higher in the patients treated with epoprostenol ( 9 of 12 patients $(75 \%)$ in the epoprostenol group vs. $\% \%$ in control group; $\mathrm{P}=0.019$; odds ratio $=24.43,95 \%$ confidence interval, 1.027-581.1] (Table). Figure is a representative image of giant fold gastritis observed in patients treated with epoprostenol. In 7 of the 9 patients with giant fold gastritis, Helicobacter pylori infection was negative on serum antibody test; the remaining patients were not tested.

The underlying cause of giant fold gastritis observed in this study could not be determined because no pathological studies have been conducted so far. And because of the small number of subjects in each group, it is unclear whether giant fold gastritis is associated with epoprostenol or not. It may be caused by other PAH-specific drugs or the patients with $\mathrm{PAH}$ happened to have giant fold gastritis before treatment. However, our results suggest that the possibility of epoprostenol causing giant fold gastritis cannot be ruled out: if patients who have been treated with epoprostenol long-term show hemodynamic exacerbations, the possibility of impaired absorption of PAH-specific oral vasodilator drugs caused by giant fold gastritis should be taken into consideration. Collaborative large studies including pathological analysis will be necessary for further research into the relationship between epoprostenol infusion and the occurrence of giant fold gastritis.

\section{References}

1. Humbert M, Sitbon O, Simonneau G. Treatment of pulmonary arterial hypertension. $N$ Engl J Med 2004; 351: 1425-1436.

2. Ogawa A, Matsubara H, Fujio H, Miyaji K, Nakamura K, Morita $\mathrm{H}$, et al. Risk of alveolar hemorrhage in patients with primary pulmonary hypertension-anticoagulation and epoprostenol therapy. Circ J 2005; 69: 216-220. 